\title{
Criminologie
}

\section{Spécificités et constantes de la culture professionnelle policière : une mise en relief de l'opinion de policiers de la région toscane}

\section{Francesco Carrer et Marc Alain}

Volume 45, numéro 1, printemps 2012

URI : https://id.erudit.org/iderudit/1008381ar

DOI : https://doi.org/10.7202/1008381ar

Aller au sommaire du numéro

Éditeur(s)

Les Presses de l’Université de Montréal

ISSN

0316-0041 (imprimé)

1492-1367 (numérique)

Découvrir la revue

Citer cet article

Carrer, F. \& Alain, M. (2012). Spécificités et constantes de la culture professionnelle policière : une mise en relief de l'opinion de policiers de la région toscane. Criminologie, 45(1), 153-180. https://doi.org/10.7202/1008381ar
Résumé de l'article

Cet article dresse un portrait sommaire du fonctionnement des organisations policières en Italie afin de situer l'organisation policière de la région de la Toscane. Nous présentons ensuite les résultats obtenus à la collecte de données des 1520 policiers de cette organisation qui se sont prêtés à l'exercice de répondre à un questionnaire anonyme destiné à mesurer, d'une part, le degré d'attachement des répondants à cinq grands thèmes de la culture professionnelle policière et, d'autre part, la propension de ces mêmes répondants quant à dénoncer ou non des comportements dérogatoires hypothétiques présentés sous forme de vignettes. Les résultats obtenus à l'analyse des réponses des policiers toscans seront comparés à ce que la passation de ce même questionnaire a pu faire ressortir dans le cas des deux études étatsuniennes qui en étaient l'origine. Cet effort de comparaison permet de faire ressortir les spécificités italiennes de même qu'un certain nombre de caractéristiques que nous pourrions attribuer à une culture professionnelle policière générale. 


\title{
Spécificités et constantes de la culture professionnelle policière: une mise en relief de l'opinion de policiers de la région toscane
}

\author{
Francesco Carrer \\ Chercheur indépendant \\ francesco.carrer@teletu.it \\ Marc Alain \\ Département de psychoéducation, Université du Québec à Trois-Rivières \\ marc.alain@uqtr.ca
}

\begin{abstract}
RÉSUMÉ - Cet article dresse un portrait sommaire du fonctionnement des organisations policières en Italie afin de situer l'organisation policière de la région de la Toscane. Nous présentons ensuite les résultats obtenus à la collecte de données des 1520 policiers de cette organisation qui se sont prêtés à l'exercice de répondre à un questionnaire anonyme destiné à mesurer, d'une part, le degré d'attachement des répondants à cinq grands thèmes de la culture professionnelle policière et, d'autre part, la propension de ces mêmes répondants quant à dénoncer ou non des comportements dérogatoires hypothétiques présentés sous forme de vignettes. Les résultats obtenus à l'analyse des réponses des policiers toscans seront comparés à ce que la passation de ce même questionnaire a pu faire ressortir dans le cas des deux études étatsuniennes qui en étaient l'origine. Cet effort de comparaison permet de faire ressortir les spécificités italiennes de même qu'un certain nombre de caractéristiques que nous pourrions attribuer à une culture professionnelle policière générale.
\end{abstract}

MOTS-CLÉS • Éthique policière, culture professionnelle policière, management de l'organisation policière, comparaisons interculturelles. 


\section{Introduction $^{1}$}

La culture cinématographique étatsunienne exploite depuis longtemps et régulièrement les icônes que constituent le flic pourri et sa contrepartie, le policier vertueux pour qui la fin ne justifie jamais les moyens. Bien évidemment, Hollywood oblige, le second finit toujours par triompher du premier. Or, si l'on veut bien s'en tenir à l'histoire relativement récente du management des organisations policières nord-américaines $^{2}$, force nous sera de reconnaitre qu'effectivement, cette histoire est marquée par des cas de corruption et de violence qui ont entraîné la mise sur pied de commissions d'enquête révélatrices de pratiques douteuses et de malversations qui s'étendaient, dans certains cas, à des pans entiers des organisations policières sous enquête.

C'est ainsi que dans le cas du New York Police Department (NYPD), par exemple, ces commissions d'enquête (en particulier la Commission Knapp, 1972) ont en quelque sorte «popularisé» le terme de pomme pourrie, bien vite devenu l'étiquette commode servant à désigner les responsables - forcément minoritaires au sein d'un groupe d'individus vertueux - de l'amorce des scandales et des dérives morales. Toujours dans le cas du NYPD, toutefois, l'une de ces commissions d'enquête, la Commission Mollen (1994), a sérieusement ébranlé la théorie voulant que c'était la pomme avariée qui contaminait le baril. C'est en effet à partir des travaux de la Commission Mollen que l'on a commencé à mettre de l'avant une hypothèse nettement plus complexe et lourde de conséquences, selon laquelle ce serait peut-être le baril lui-même qui faisait pourrir les pommes. Dès lors, bien sûr, la réflexion éthique allait prendre une tout autre tournure. Et à cet égard, comme nous le mentionnions dans le cas du Québec, par exemple (Alain, 2004), la question éthique est progressivement devenue un élément incontournable de la réflexion sur le fonctionnement de ces organisations complexes que sont devenues les forces policières contemporaines.

1. Les auteurs tiennent à remercier pour leur collaboration et leur très grande ouverture les autorités du secteur des Politiques pour la sécurité urbaine de la région toscane qui ont rendu possible cette recherche; il va cependant de soi que les opinions et analyses présentées ici n'engagent que les auteurs.

2. Il convient de rappeler ici que, contrairement à plusieurs pays européens et sudaméricains, les États-Unis et le Canada ne disposent d'organisations policières vraiment structurées que depuis la fin du XIX ${ }^{e}$ siècle et c'est particulièrement le cas dans les grandes villes. 
L'Europe n'a pas elle non plus complètement échappé à cet enjeu. Il convient toutefois de relever à quel point l'exercice est demeuré essentiellement britannique. Jamais, par exemple, la France n'a mis sur pied une commission indépendante et externe pour juger de situations problématiques ayant éclaté au grand jour ${ }^{3}$. Le cas de la Belgique est à peu près similaire, les travaux parlementaires faisant suite à l'affaire Dutroux de 1998 ayant été en partie justifiés par la révélation de l'existence d'une «guerre des polices» belges (Alain, 2000, 2001) ${ }^{4}$. On notera donc, pour en revenir au contexte anglo-saxon, les rapports Scarman (1992), Macpherson (1999) et, dans le contexte plus spécifique à la gestion du système policier irlandais, le rapport Pattern (1999) et le rapport O'Loan (2001). Dans les deux premiers cas, ce sont essentiellement des incidents à caractère raciste impliquant des policiers qui ont conduit à la mise sous enquête, tandis que dans les deux autres, on évoquait plutôt des manquements en matière de limites à l'emploi de la force et de technologies répressives. Les conclusions des rapports Scarman et Macpherson mettaient en exergue l'importance d'accentuer les politiques d'ouverture et d'établissement de liens de confiance entre la police et les représentants des minorités, tandis que les rapports Pattern et O'Lean, quant à eux, réitéraient l'importance du respect des droits de la personne comme garantie d'une meilleure collaboration avec les communautés.

À l'instar des autres pays de l'Europe continentale, l'Italie n'échappe pas vraiment non plus au constat général d'une quasi-absence du recours à des modalités d'enquête externes et indépendantes sur le fonctionnement des organisations policières. En ce qui a trait à la Police d'État (la Polizia di Stato, le corps de police civile le plus important au pays), deux mécanismes sont à la disposition des autorités: il s'agit des commissions d'enquête dites «techniques», dont les mandats et les membres sont nommés par le chef de la Police d'État, et les commissions dites «politiques» qui sont, quant à elles, sous la responsabilité du Parlement. Dans le premier cas, les rapports sont remis directement au directeur, qui décide alors et en toute autonomie s'il les rend publics ou non. Quelques-uns de ces rapports émergent toutefois de temps en temps;

3. Comme ce fut notamment le cas des événements entourant les grandes émeutes de 2005 dans les banlieues.

4. C'est d'ailleurs cette affaire qui a conduit la Belgique à réformer en profondeur l'ensemble de son système policier. 
citons le cas de l'enquête du préfet Serra, en 1995, qui tournait autour de l'affaire de «la bande de la Uno Bianca ${ }^{5}$ », ou bien ceux, plus récents, des enquêtes du préfet Micalizio et des directeurs Montanaro et Cernetig, rapports qui faisaient la lumière sur les désordres ayant entouré la tenue du Sommet du G8 à Gênes en 2001.

Les commissions d'enquête parlementaires, quant à elles, sont formées d'élus qui représentent les partis au Parlement, soit le parti majoritaire et les partis minoritaires. Ce mécanisme fait toutefois en sorte que plusieurs rapports différents peuvent être produits sur les mêmes événements, l'un représentant l'opinion des membres du parti au pouvoir, le ou les autres, celle des membres de l'opposition. Les policiers pourront, à la suite des révélations de ces commissions, faire l'objet, comme tout citoyen, de plaintes et de poursuites par les autorités judiciaires. En pratique, toutefois, la garantie qu'ils puissent rectifier leur comportement par le biais de la dissuasion judiciaire demeure assez théorique. Il importe, tout d'abord, que le comportement dérogatoire soit considéré comme un délit au sens de la loi par les trois paliers de justice existants - en Italie, une condamnation définitive arrive généralement après que se soient prononcés les juges de trois paliers différents, soit, ceux du Tribunal ordinaire, de la Cour d'appel et de la Cour de cassation. Mais en admettant que ce soit le cas, il pourra s'écouler une dizaine d'années entre la dénonciation et le prononcé d'un verdict, une période au cours de laquelle le policier pourra généralement poursuivre ses activités en ayant parfois à subir, tout au plus, qu'une mutation dans un territoire éloigné de quelques kilomètres de celui de son assignation d'origine. Et c'est sans compter le mécanisme dit du «patteggia$m e n t 0^{6} »$, faisant en sorte qu'en admettant sa culpabilité contre réduction de peine, n'importe quel accusé, et donc même le policier reconnu coupable, pourra très vite côtoyer à nouveau le citoyen qui l'avait dénoncé. De manière générale, donc, on plaide systématiquement le «coup de folie» ou «la pomme pourrie» sans qu'une réflexion en profondeur sur le fonctionnement global de l'appareil policier en cause ne soit enclenchée.

5. L'affaire révéla l'existence d'un groupe de policiers de la Polizia di Stato qui, de 1987 à 1994, effectuèrent une centaine de hold-up et d'assauts au fusil automatique en tuant 24 personnes, y compris des carabiniers, et en blessant une centaine d'autres, avant d'être arrêtés.

6. Un mécanisme introduit en 2003 afin de tenter d'accélérer le processus judiciaire. 


\section{Les forces de police en Italie}

Avant d'en arriver plus spécifiquement aux éléments relatifs à l'échantillonnage, à la méthodologie et aux résultats de nos démarches de recherche, nous nous devons de situer ces mêmes résultats dans le contexte de l'aménagement des différentes forces de police en Italie, de même que leurs spécificités intrinsèques. Ce faisant, le lecteur sera ainsi mieux en mesure de situer nos résultats et les discussions qui les sous-tendent dans ce grand ensemble quelque peu hétéroclite (Carrer, 2004, 2006). On distingue en Italie deux forces de police à compétence générale que l'on retrouve à la grandeur du pays, soit la Police d'État et les Carabiniers (une police civile dans le premier cas, et militaire dans le second), avec des effectifs de 100000 et 116000 officiers respectivement. On retrouve également, toujours sur le plan national, plusieurs corps de police à vocation plus spécifique, soit: la Garde des finances (police à statut militaire qui relève des autorités ministérielles responsables des finances de l'État, une force composée de 67000 officiers); la Police pénitentiaire (32 000 officiers); le Corps forestier de l'État (10 000 officiers); et, finalement, la Garde côtière (11 000 officiers qui sont intégrés à la marine militaire).

On doit comprendre ici que cette énumération n'a pas qu'une simple valeur encyclopédique; en effet, si, en apparence, les mandats et spécificités des missions semblent relativement clairs, sur le terrain, les choses le sont beaucoup moins. Les opérations de maintien de l'ordre, par exemple, peuvent mobiliser plusieurs forces de police simultanément dans des situations où les compétences des uns et des autres se superposent. De manière analogue, et tel qu'il est prévu à l'article $12 \mathrm{du}$ Code de la route, un conducteur automobile peut faire l'objet d'un contrôle routier par la Police routière - une des branches de la Police d'État mais tout aussi bien par l'une ou l'autre des autres forces de police italiennes, qu'il s'agisse d'une police municipale, des Carabiniers, de la Garde des finances, de la Police pénitentiaire, voire du Corps forestier. À l'exception de quelques activités de formation suivies en commun et limitées à des aspects juridiques très spécifiques, chaque force de police en présence sur le territoire italien effectue son recrutement, sa sélection et sa formation (de base et continue) de manière totalement autonome et indépendante. Cette autonomie et indépendance des corps policiers les uns par rapport aux autres ne manque pas d'influencer, en Italie comme dans plusieurs autres pays (Alain, 2000), le climat de collaboration et de coopération. Par ailleurs, il est clair que les fonctionnements policiers 
vont varier considérablement d'une administration à une autre, d'une région à une autre, voire d'un corps de police à un autre au sein d'une même localité. À tout cela, ajoutons également que chaque corps de police dispose d'une tradition, d'une culture, d'une histoire de ses relations avec les élus et la population qui lui est propre, ce qui rend d'autant plus difficile tout exercice de généralisation. En raison de ce contexte, les sensibilités aux dilemmes éthiques varient, elles aussi, considérablement dans toute l'Italie. C'est donc en fonction de ces réalités qu'il est à peu près impossible de penser que le cas de la police de la région toscane que nous allons maintenant aborder est représentatif de la situation nationale italienne de quelque façon que ce soit.

\section{Contexte, échantillonnage et méthodologie}

Sondages, valeurs et culture de l'organisation policière

Bien que la question de la culture professionnelle des policiers constitue un pan de recherche important et qui remonte aux toutes premières réflexions sociologiques sur cette institution (Bitner, 1966, 1967a, 1967b), on considère que ce sont Milton Rokeach et ses collègues (1971) qui ont été les premiers à utiliser le sondage comme outil privilégié pour assurer ce travail. Les gestionnaires des organisations policières se sont vite rendu compte qu'en sondant les policiers de leurs organisations, la planification de changements importants s'en trouvait facilitée; on peut évoquer ici, par exemple, le fait de passer d'une prestation de services plus ou moins classique à l'une ou l'autre des modalités de police communautaire (Greene et al., 1994). Mais ces efforts et les résultats obtenus demeurent généralement inconnus des chercheurs et réservés aux seules fins de prises de décisions administratives, sans compter que ces résultats ne représentent pas nécessairement les attitudes d'échantillons représentatifs stratifiés de la population policière d'un État ou d'une région donnée. C'est précisément à ce genre de problèmes que se sont attaqués Weisburd et son équipe (2000). L'outil développé dans le cadre de cette étude comporte 92 questions dont 23 peuvent faire l'objet de regroupements qui touchent divers aspects du problème de l'abus d'autorité par la police?

7. Ces thèmes sont: (1) l'attitude générale face à l'emploi de la force; (2) l'importance accordée au code du silence; (3) l'influence du statut socioéconomique des personnes arrêtées 
Weisburd et al. (2001) ont montré que les répondants qui pensent que les abus d'autorité sont liés à des politiques moins tolérantes sont également ceux qui croient que dans certains cas, ces abus sont acceptables et ne devraient pas faire l'objet d'une dénonciation entre collègues, ou d'un policier à son supérieur hiérarchique. Si le questionnaire créé par Weisburd permet une compréhension du positionnement culturel des répondants, celui élaboré indépendamment par Klockars et al. (1997) nous permet de faire avancer cette compréhension d'une manière un peu différente. Ce dernier questionnaire a déjà fait l'objet de plusieurs études de comparaisons internationales (Ivkovich et Klockars, 1995; Punch et al., 2000; Klockars et al., 2003). Dans ce cas-ci, on demande aux répondants de porter un jugement sur 11 scénarios de comportements dérogatoires, ou vignettes, en fonction de sept dimensions différentes. On demande au répondant de juger (1) de la gravité du comportement décrit dans la vignette (sur une échelle à cinq points, allant de «pas du tout grave» à «extrêmement grave»), (2) de la manière dont il croit que ses collègues et (3) son organisation jugeraient le comportement (la même échelle que précédemment). Ensuite, (4) le répondant doit émettre une opinion quant à sanctionner ou non le comportement et, s'il préconise une sanction, dans quelle mesure. (Il doit également faire la même chose pour ce qu'il pense (5) que ses collègues et (6) son organisation émettraient en termes de sanction et ce, sur une échelle à six points, allant de «aucune sanction» jusqu'à «destitution»). Finalement, (7) on demande au répondant s'il dénoncerait le comportement, sur une échelle à cinq points, allant de «absolument pas» à «absolument».

\section{Population et échantillon dans la région toscane}

Les travaux de terrain liés à cette recherche ont été menés sous l'égide du secteur des Politiques pour la sécurité urbaine de la région toscane. L'ensemble des personnels policiers des polices locales de la région a été approché; il s'agit, dans les faits, des corps de police qui dépendent d'administrations locales de taille moyenne, comme c'est le cas des villes de Florence, Pise, Sienne et Livorno, mais aussi de plus petites administrations municipales et de communes. C'est donc un effectif total

sur l'action des policiers; (4) l'effet des mesures destinées à atténuer les abus d'autorité; et (5) les effets de la police communautaire sur le phénomène de l'abus d'autorité. 
de 4017 policiers et policières qui constituait, au moment de l'enquête, l'ensemble de la population à l'étude. Après en avoir distribué suffisamment pour être certain de rejoindre l'ensemble de ces policiers, 1553 questionnaires $^{8}$ nous ont été retournés; l'opération, du début de la distribution jusqu'à la fin de la collecte s'est étendue sur neuf mois, de juin 2006 à février 2007. De ces questionnaires, 33 étaient vierges ou inutilisables. Nous pouvons donc compter sur les réponses de 1520 de ces policiers, pour un taux de réponse de près de $38 \%$. En termes strictement statistiques, et selon l'équation de Sudman $(1976)^{9}$, ces 1520 réponses valides sont globalement représentatives de la population agrégée. Par contre, et comme le montrent les chiffres du tableau suivant, on constatera que la répartition des répondants selon leur niveau hiérarchique n'est pas représentative; compte tenu du fait que les agents de premier niveau ont été moins portés à répondre que leurs supérieurs, nous devrons pondérer les résultats descriptifs en fonction de cet élément.

\section{Résultats}

Nous présenterons les résultats obtenus en comparant trois échantillons différents. Il s'agit tout d'abord des deux échantillons étatsuniens attachés à chacun des deux questionnaires utilisés (dans le cas de l'Italie, les deux questionnaires étaient présentés l'un à la suite de l'autre aux répondants). Pour l'étude menée par Weisburd et son équipe, ce sont 925 officiers de police provenant de 121 organisations différentes qui se sont prêtés à l'exercice, tandis que pour la recherche menée par Klockars et al. (1997), il s'agit d'un échantillon de 3235 policiers, provenant de 30 agences de police différentes. Finalement, le troisième échantillon est celui issu des policiers de la région toscane, tel que nous l'avons décrit dans la section précédente.

8. Chaque questionnaire était accompagné d'une lettre expliquant les modalités et les objectifs de la démarche, ainsi qu'une garantie du respect de l'anonymat des répondants.

9. Cette équation, $n=\frac{N}{1+N(e)^{2}}$, génère des bornes minimales d'échantillons statistiquement représentatifs à 5 pour cent ou moins (soit le terme $e$ de l'équation en question). Dans le cas qui nous concerne ici, et dans le cas d'une passation strictement aléatoire des questionnaires auprès de ces 4017 policiers, un échantillon de 364 répondants aurait constitué la borne minimale de représentativité à un seuil de confiance de $95 \%$. 
TAB LEA U 1

Les trois caractéristiques sociodémographiques, selon l'échantillon et la population de référence des policiers de la région toscane

\begin{tabular}{|l|c|r|r|r|}
\hline & \multicolumn{2}{|c|}{ Population $(\mathrm{n}=4017)$} & \multicolumn{2}{c|}{ Échantillon $(\mathrm{n}=1520)$} \\
\hline Le genre: & $\mathrm{n}$ & $\%$ & $\mathrm{n}$ & $\%$ \\
Femmes & 1485 & 37,0 & 410 & 29,9 \\
Hommes & 2532 & 63,0 & 959 & 70,1 \\
Total valide & $\mathbf{4 0 1 7}$ & $\mathbf{1 0 0 , 0}$ & $\mathbf{1 3 6 9}$ & $\mathbf{1 0 0 , 0}$ \\
Les groupes d'âge: & & & & \\
$20-30$ ans & 318 & 7,9 & 112 & 9,2 \\
$31-40$ ans & 1289 & 32,1 & 400 & 32,7 \\
$41-50$ ans & 1567 & 39,0 & 480 & 39,3 \\
$51-60$ ans & 558 & 13,9 & 219 & 17,9 \\
61 ans et plus & 285 & 7,1 & 11 & 0,9 \\
Total valide & 4017 & 100,0 & 1222 & 100,0 \\
Les niveaux hiérarchiques: & & & & \\
Agents & 3080 & 76,7 & 902 & 66,3 \\
Sous-officiers & 839 & 20,9 & 435 & 31,9 \\
Officiers & 98 & 2,4 & 23 & 1,8 \\
Total valide & 4017 & 100,0 & 1360 & 100,0 \\
\hline
\end{tabular}

Source: Secteur des Politiques pour la sécurité urbaine de la région toscane, enquête administrative de 2005-2006.

Les rapports à l'abus d'autorité, une réplique des travaux de Weisburd et al. (2000)

L'essence de l'effort descriptif et comparatif auquel nous allons maintenant nous atteler consiste à voir jusqu'à quel point on pourra ou non déceler certains traits culturels, certaines attitudes qui semblent transcender les deux pays d'origine des répondants. Il s'agit, en d'autres termes, de voir jusqu'à quel point nous serons ou non en mesure de démontrer l'existence d'une trame culturelle professionnelle policière plus ou moins universelle. En supposant qu'une telle universalité ne se retrouve pas comme telle, ce sont alors plutôt les écarts et leur ampleur, selon les cinq grands thèmes touchés, que nous serons en mesure de commenter. Comme l'avaient préalablement fait Weisburd et al. (2000), nous présenterons chacun de ces thèmes les uns à la suite des autres plutôt qu'en un seul bloc. Pour les prochains tableaux, la première ligne correspond à l'échantillon original des policiers étatsuniens (Weisburd et al., 2000) et la seconde, à l'échantillon de policiers italiens (en caractères soulignés). Pour ces deux échantillons, les fréquences présentées sont pondérées afin de tenir compte des différences de paramètres entre ces échantillons et les populations dont ils sont tirés. 
TAB LEA U 2

Attitudes générales face à l'usage de la force, en pourcentages des réponses données par les deux échantillons

\begin{tabular}{|l|c|c|c|c|}
\hline & $\begin{array}{c}\text { Totalement } \\
\text { d'accord }\end{array}$ & D'accord & $\begin{array}{c}\text { En } \\
\text { désaccord }\end{array}$ & $\begin{array}{c}\text { Totalement } \\
\text { en désaccord }\end{array}$ \\
\hline $\begin{array}{l}\text { On ne permet pas à la } \\
\text { police d'utiliser autant } \\
\text { de force qu'il est souvent } \\
\text { nécessaire lors des } \\
\text { arrestations. }\end{array}$ & 6,2 & 24,9 & 60,5 & 8,4 \\
\hline $\begin{array}{l}\text { Il est parfois acceptable } \\
\text { d'utiliser plus de force } \\
\text { qu'il n'est légalement } \\
\text { permis de le faire afin } \\
\text { de contrôler une personne } \\
\text { qui s'en prend physique- } \\
\text { ment à un autre policier. }\end{array}$ & $\underline{33,3}$ & $\underline{26,7}$ & $\underline{21,1}$ & $\underline{18,8}$ \\
\hline $\begin{array}{l}\text { Suivre toujours les } \\
\text { règlements n'est pas } \\
\text { compatible avec faire } \\
\text { le travail. }\end{array}$ & $\underline{31,5}$ & $\underline{21,5}$ & $\underline{23,6}$ & $\underline{20,0}$ \\
\hline
\end{tabular}

Entre les trois questions qui composent ce thème, on observe que les opinions des deux échantillons sont assez différentes pour les deux premières et particulièrement proches pour la troisième. Pour ce qui est des deux premières questions, comme dans quelques autres que nous verrons plus bas, les répondants étatsuniens se situent pratiquement à l'opposé de leurs collègues italiens. De manière générale, les réponses données par les répondants étatsuniens vont dans le sens des réponses attendues par les tenants d'une vision, politiquement correcte dirionsnous, de la police. Si tant est que le même phénomène tend à se répéter régulièrement, il y aura lieu d'invoquer l'hypothèse d'un phénomène d'hétéroduperie $^{10}$ (Tournois et al., 2000). De manière un peu plus

10. L'hétéroduperie fait référence au concept de désirabilité sociale dans la manière que peuvent avoir des répondants de répondre non pas tellement selon ce qu'ils pensent, mais bien en fonction de ce qu'ils pensent que les sondeurs attendent d'eux. Des échanges de courriers électroniques avec le premier auteur de l'étude étatsunienne (i. e. David Weisburd) nous ont permis d'apprendre que cet effet n'avait pas fait l'objet de vérifications plus pointues lors de la passation du questionnaire en sol étatsunien. Comme nous ne l'avons pas fait non plus, nous posons que toute chose demeure à peu près équivalente à cet égard dans l'autre échantillon. Ceci dit, il est clair qu'un incident de l'ampleur de l'affaire Rodney King, pour ne prendre que cet exemple, pourra très certainement avoir un effet non négligeable sur l'hétéroduperie; dans le cas de l'Italie, à tout le moins, nous savons qu'au moment où a eu lieu la passation, le paysage médiatique était libre de ce genre d'affaires. 
spécifique, il convient de remarquer que les policiers de la région toscane ne sont armés que d'un pistolet et d'une bonbonne de poivre de Cayenne et qu'ils ne peuvent donc pas recourir à l'ensemble de la panoplie des armes à létalité atténuée, des armes dont la quasi-totalité des policiers étatsuniens sont dotés et dont ils ont une disponibilité immédiate ${ }^{11}$. Considérant ces éléments, il est probablement «facile» pour les répondants étatsuniens de déclarer qu'on leur permet effectivement d'utiliser autant de force que nécessaire, ce qui n'est pas le cas de leurs collègues italiens, contraints à une panoplie technique beaucoup plus réduite. Dans le cas de la troisième question, Italiens et Étatsuniens tendent toutefois à répondre dans le sens attendu, i. e. en reconnaissant en majorité être en désaccord avec l'affirmation liée au fait que suivre les règlements n'est pas compatible avec faire le travail. On notera, toutefois, que si les répondants étatsuniens, toujours pour la troisième question de ce thème, émettent des opinions plus concentrées autour d'un hypothétique point neutre (i. e., entre «D'accord» et «En désaccord»), les répondants italiens, en revanche, hésitent moins à faire part d'opinions plus aux deux extrêmes. Cette différence, encore une fois, pourrait refléter le fait que les répondants italiens ont, un peu moins que leurs collègues étatsuniens, tendance à répondre dans «le sens attendu».

Les réponses données aux cinq questions du tableau 3 illustrent sans contredit des différences de cultures organisationnelles marquées. Dans le cas de la première question, et bien que les répondants italiens expriment majoritairement être en désaccord avec l'affirmation que le code du silence est une partie essentielle de la confiance mutuelle entre collègues, on notera toutefois que la réponse de cette majorité - $60 \%$ - est tout de même moins franche que celle fournie par les répondants étatsuniens (à $83 \%$ ). Les quatre autres questions de ce tableau renvoient à la dénonciation; or, ici, il y a quasi-homogénéité des réponses des deux échantillons à deux questions et une franche opposition dans les deux autres. Si l'on commence par les deux questions sur lesquelles il y a cohésion - la seconde et la cinquième -, on fait ici appel à la notion

11. Une autre explication potentielle, que nous a très aimablement suggérée l'un des évaluateurs, consisterait en ceci que les policiers italiens travaillent dans un contexte nettement plus réglementé et moins négociable que leurs confrères étatsuniens qui, eux, travaillent davantage dans une logique jurisprudentielle et pragmatique, dont on peut effectivement penser qu'elle favorise une certaine souplesse dans l'adaptation des règles aux réalités. 
TABLEAU 3

Le thème du «code du silence », en pourcentages des réponses données par les deux échantillons

\begin{tabular}{|c|c|c|c|c|}
\hline & $\begin{array}{l}\text { Totalement } \\
\text { d'accord }\end{array}$ & D'accord & $\begin{array}{c}\text { En } \\
\text { désaccord }\end{array}$ & $\begin{array}{l}\text { Totalement } \\
\text { en désaccord }\end{array}$ \\
\hline $\begin{array}{l}\text { Le code du silence est } \\
\text { une partie essentielle de } \\
\text { la confiance mutuelle } \\
\text { nécessaire au bon travail } \\
\text { policier. }\end{array}$ & $\begin{array}{r}1,2 \\
21,3 \\
\end{array}$ & $\begin{array}{l}15,7 \\
18,6 \\
\end{array}$ & $\begin{array}{l}65,6 \\
\underline{31,1} \\
\end{array}$ & $\begin{array}{l}17,5 \\
29,0 \\
\end{array}$ \\
\hline $\begin{array}{l}\text { Il ne vaut pas le coup, } \\
\text { pour le dénonciateur, de } \\
\text { dénoncer tout problème. }\end{array}$ & $\begin{array}{r}3,1 \\
13,8 \\
\end{array}$ & $\begin{array}{l}21,8 \\
14,5 \\
\end{array}$ & $\begin{array}{l}63,5 \\
\underline{35,1} \\
\end{array}$ & $\begin{array}{l}11,7 \\
\underline{36,6} \\
\end{array}$ \\
\hline $\begin{array}{l}\text { Un policier qui dénonce } \\
\text { les agissements d'un } \\
\text { collègue risque d'être } \\
\text { rejeté par les autres } \\
\text { policiers. }\end{array}$ & $\begin{array}{r}11,0 \\
14,7 \\
\end{array}$ & $\begin{array}{r}56,4 \\
19,2\end{array}$ & $\begin{array}{l}30,9 \\
\underline{30,8} \\
\end{array}$ & $\begin{array}{r}1,8 \\
35,3 \\
\end{array}$ \\
\hline $\begin{array}{l}\text { Il est courant pour un } \\
\text { policier d'ignorer la } \\
\text { conduite incorrecte } \\
\text { d'autres policiers. }\end{array}$ & $\begin{array}{l}1,8 \\
\underline{3,9}\end{array}$ & $\begin{array}{r}50,6 \\
\underline{4,3}\end{array}$ & $\begin{array}{l}43,3 \\
\underline{38,6} \\
\end{array}$ & $\begin{array}{r}4,4 \\
53,2 \\
\end{array}$ \\
\hline $\begin{array}{l}\text { En contexte d'abus } \\
\text { d'autorité commis par } \\
\text { d'autres policiers, } \\
\text { les policiers rapportent } \\
\text { toujours les cas de } \\
\text { violation criminelle grave. }\end{array}$ & $\begin{array}{r}2,8 \\
14,6 \\
\end{array}$ & $\begin{array}{l}36,2 \\
25,6 \\
\end{array}$ & $\begin{array}{l}58,5 \\
26,0 \\
\end{array}$ & $\begin{array}{r}2,5 \\
33,9 \\
\end{array}$ \\
\hline
\end{tabular}

de dénoncer une dérogation pour en quelque sorte mieux faire fonctionner l'organisation; il s'agit donc, en extrapolant, du principe même de l'utilité de la dénonciation. Les deux questions où il y a opposition, par contre, font état de la principale conséquence appréhendée du fait de dénoncer un comportement problématique, soit de se sentir rejeté par les autres. Selon le sens du pourcentage des réponses, il appert ici que les répondants étatsuniens, et contrairement à ce que nous avions constaté au tableau précédent, expriment une opinion carrément contraire au «sens attendu». En d'autres termes, les répondants italiens semblent montrer un peu plus de cohérence entre le principe du code du silence - en le rejetant; première question du tableau 3 - et le fait que leurs collègues n'hésitent pas à effectivement dénoncer une conduite incorrecte (quatrième question). Plus globalement, ces réponses indiquent un clivage significatif, chez les policiers étatsuniens, entre, d'une 
part, les politiques organisationnelles officielles et, d'autre part, l'importance de la solidarité entre collègues; un clivage qui se trouve être beaucoup moins marqué chez nos répondants italiens.

TA B LEA U 4

Les impacts de l'attitude, de l'origine ethnique et du statut socioéconomique des citoyens sur le comportement policier, en pourcentages des réponses données par les deux échantillons

\begin{tabular}{|c|c|c|c|c|}
\hline & $\begin{array}{c}\text { Totalement } \\
\text { d'accord }\end{array}$ & D'accord & $\begin{array}{c}\text { En } \\
\text { désaccord }\end{array}$ & $\begin{array}{l}\text { Totalement } \\
\text { en désaccord }\end{array}$ \\
\hline $\begin{array}{l}\text { Un policier est plus } \\
\text { susceptible d'arrêter une } \\
\text { personne qui, selon lui, } \\
\text { fait montre d'une } \\
\text { mauvaise attitude. }\end{array}$ & $\begin{array}{r}2,1 \\
20,1 \\
\end{array}$ & $\begin{array}{r}46,7 \\
32,9 \\
\end{array}$ & $\begin{array}{l}45,1 \\
24,0 \\
\end{array}$ & $\begin{array}{r}6,1 \\
23,1 \\
\end{array}$ \\
\hline $\begin{array}{l}\text { Les policiers traitent } \\
\text { souvent mieux les Blancs } \\
\text { que les personnes issues } \\
\text { des minorités visibles. }\end{array}$ & $\begin{array}{r}1,2 \\
10,1 \\
\end{array}$ & $\begin{array}{l}15,8 \\
14,4\end{array}$ & $\begin{array}{r}57,8 \\
27,2\end{array}$ & $\begin{array}{l}25,2 \\
48,3\end{array}$ \\
\hline $\begin{array}{l}\text { Les policiers sont plus } \\
\text { susceptibles de recourir } \\
\text { à l'usage de la force à } \\
\text { l'endroit de personnes } \\
\text { issues de minorités } \\
\text { visibles qu'à l'endroit } \\
\text { de Blancs dans des } \\
\text { situations similaires. }\end{array}$ & $\begin{array}{r}1,7 \\
11,5 \\
\end{array}$ & $\begin{array}{r}9,4 \\
16,1 \\
\end{array}$ & $\begin{array}{r}55,6 \\
30,6 \\
\end{array}$ & $\begin{array}{r}33,3 \\
41,8\end{array}$ \\
\hline $\begin{array}{l}\text { Les policiers sont plus } \\
\text { susceptibles de recourir } \\
\text { à l'usage de la force à } \\
\text { l'endroit de personnes } \\
\text { pauvres qu'à l'endroit } \\
\text { de personnes des classes } \\
\text { moyennes dans des } \\
\text { situations similaires. }\end{array}$ & $\begin{array}{l}1,9 \\
\underline{8,3}\end{array}$ & $\begin{array}{r}12,3 \\
11,9\end{array}$ & $\begin{array}{l}57,9 \\
26,8\end{array}$ & $\begin{array}{r}27,9 \\
53,0 \\
\end{array}$ \\
\hline
\end{tabular}

Les réponses que nous ont données ici les répondants italiens ne se démarquent pas aussi franchement que ce que nous avons constaté plus haut: bien qu'avec légèrement moins de conviction que les répondants étatsuniens (surtout à la troisième question), Italiens et Étatsuniens s'entendent à penser que les caractéristiques des citoyens ne doivent pas être des motifs d'intervention différenciée. En revanche, la question de l'attitude du citoyen - première question - donne, elle, des résultats plus nuancés; les réponses dans le sens favorable ou défavorable des 
TABLEAU 5

La perception quant aux modalités de contrôle organisationnel des abus d'autorité, en pourcentages des réponses données par les deux échantillons

\begin{tabular}{|c|c|c|c|c|}
\hline & $\begin{array}{c}\text { Totalement } \\
\text { d'accord }\end{array}$ & D'accord & $\begin{array}{c}\text { En } \\
\text { désaccord }\end{array}$ & $\begin{array}{l}\text { Totalement } \\
\text { en désaccord }\end{array}$ \\
\hline $\begin{array}{l}\text { Votre organisation a pris } \\
\text { une position très ferme } \\
\text { face aux comportements } \\
\text { incorrects des policiers. }\end{array}$ & $\begin{array}{r}35,2 \\
43,5 \\
\end{array}$ & $\begin{array}{r}57,4 \\
29,1\end{array}$ & $\begin{array}{r}6,6 \\
15,8 \\
\end{array}$ & $\begin{array}{r}0,9 \\
11,6 \\
\end{array}$ \\
\hline $\begin{array}{l}\text { Les enquêtes sur les } \\
\text { agissements incorrects } \\
\text { des policiers sont } \\
\text { généralement biaisées en } \\
\text { faveur des policiers. }\end{array}$ & $\begin{array}{r}0,4 \\
24,7 \\
\end{array}$ & $\begin{array}{r}5,1 \\
35,2 \\
\end{array}$ & $\begin{array}{l}72,4 \\
\underline{21,3} \\
\end{array}$ & $\begin{array}{l}22,0 \\
18,7 \\
\end{array}$ \\
\hline $\begin{array}{l}\text { Si le chef de l'organisa- } \\
\text { tion prend une position } \\
\text { très ferme face aux abus } \\
\text { d'autorité, il peut } \\
\text { contribuer à ce que les } \\
\text { policiers n'abusent pas de } \\
\text { leur autorité. }\end{array}$ & $\begin{array}{l}24,5 \\
\underline{64,9} \\
\end{array}$ & $\begin{array}{l}60,3 \\
28,2 \\
\end{array}$ & $\begin{array}{r}13,8 \\
\underline{3,5} \\
\end{array}$ & $\begin{array}{l}1,4 \\
\underline{3,4} \\
\end{array}$ \\
\hline $\begin{array}{l}\text { Les bons superviseurs } \\
\text { peuvent aider à prévenir } \\
\text { les abus d'autorité des } \\
\text { policiers. }\end{array}$ & $\begin{array}{l}22,9 \\
\underline{56,0} \\
\end{array}$ & $\begin{array}{l}66,9 \\
34,4 \\
\end{array}$ & $\begin{array}{l}9,3 \\
\underline{5,6}\end{array}$ & $\begin{array}{l}0,9 \\
4,0 \\
\end{array}$ \\
\hline $\begin{array}{l}\text { La plupart des situations } \\
\text { d'abus d'autorité de la } \\
\text { part des policiers } \\
\text { pourraient être évitées } \\
\text { par des méthodes de } \\
\text { supervision plus efficaces. }\end{array}$ & $\begin{array}{r}7,3 \\
47,1 \\
\end{array}$ & $\begin{array}{r}48,0 \\
37,0 \\
\end{array}$ & $\begin{array}{r}39,5 \\
\underline{9,0}\end{array}$ & $\begin{array}{l}5,2 \\
\underline{7,0}\end{array}$ \\
\hline & \multicolumn{2}{|c|}{ Oui } & \multicolumn{2}{|r|}{ Non } \\
\hline $\begin{array}{l}\text { Pensez-vous qu'une } \\
\text { formation en éthique } \\
\text { permet de prévenir l'abus } \\
\text { d'autorité? }\end{array}$ & \multicolumn{2}{|c|}{$\underline{84,8}$} & \multicolumn{2}{|c|}{15,2} \\
\hline $\begin{array}{l}\text { Pensez-vous qu'une } \\
\text { formation en relations } \\
\text { interpersonnelles permet } \\
\text { de prévenir l'abus? }\end{array}$ & \multicolumn{2}{|c|}{80,3} & & $\begin{array}{l}19,7 \\
16,0 \\
\end{array}$ \\
\hline $\begin{array}{l}\text { Pensez-vous qu'une } \\
\text { formation en sensibilisa- } \\
\text { tion aux diversités } \\
\text { culturelles et humaines } \\
\text { permet de prévenir l'abus } \\
\text { d'autorité? }\end{array}$ & \multicolumn{2}{|c|}{$\underline{82,6}$} & \multicolumn{2}{|r|}{$\underline{17,4}$} \\
\hline
\end{tabular}


répondants des deux échantillons sont sensiblement les mêmes, mais les Étatsuniens se situent un peu plus au centre que les Italiens dans leurs réponses.

Les premières questions du tableau 5 révèlent des différences d'opinion qui tournent essentiellement autour d'éléments organisationnels. On constatera ici que les répondants italiens se montrent un peu plus sceptiques que les Étatsuniens, particulièrement à la deuxième question, en ce qui concerne le traitement réservé par leur organisation aux policiers dont les comportements ont été incorrects. Par contre, comme l'illustrent les réponses données à la troisième question, il est clair que ces mêmes répondants italiens souhaiteraient une volonté organisationnelle plus affirmée. Par ailleurs, on remarquera, dans le cas des trois dernières questions reliées à la formation en emploi, et peu importe le thème, que tous démontrent une attitude très ouverte à l'idée que la formation continue puisse avoir des impacts positifs. Pour en revenir à la question des modalités de contrôle organisationnel, et sans que nous ayons une connaissance précise du fonctionnement des organisations étatsuniennes à cet égard, le fait est que dans le région toscane, la hiérarchie, d'une part, exerce un contrôle important et assez direct tandis qu'elle est, d'autre part, très proche des policiers de la base. On s'y sent donc plus contrôlé, certes, mais on sent également que ce contrôle est somme toute assez impartialement exercé.

On peut observer ici, en fonction des trois possibilités de réponses, des similitudes dans les opinions et des disparités marquées et ce, pour chacune des trois questions. On observe tout d'abord une forte ressemblance des réponses quant au fait que le modèle de police communautaire ne fait pas augmenter les problèmes d'abus et de corruption, ce qui fait, reconnaissons-le, un certain sens. Par contre, quant à savoir jusqu'à quel point l'introduction de ce modèle de service a eu ou non un impact positif, là, les répondants italiens font montre de beaucoup moins d'enthousiasme que leur contrepartie étatsunienne. Sans avoir la possibilité ici de vérifier plus avant cette assertion, il faudrait en comprendre que les expériences du modèle de police communautaire au quotidien sont probablement très différentes dans des contextes socioéconomiques et politiques qui le sont tout autant.

On retiendra, au final de cet exercice, que les perceptions des répondants sur quelques grandes thématiques de la culture et de l'organisation policière sont parfois assez disparates et parfois beaucoup moins, d'un thème à un autre et d'un pays à un autre. Très globalement, il en ressort 
TAB LEA U 6

Le modèle de police communautaire et ses effets sur la question des abus d'autorité de la part de la police, en pourcentages des réponses données par les deux échantillons

\begin{tabular}{|l|c|c|c|}
\hline & Augmenter & Diminuer & $\begin{array}{c}\text { N'a pas eu } \\
\text { d'impact }\end{array}$ \\
\hline $\begin{array}{l}\text { Pensez-vous que la police de type } \\
\text { communautaire a fait augmenter, } \\
\text { diminuer ou n'a eu aucun impact sur } \\
\text { les problèmes de corruption policière? }\end{array}$ & $\underline{8,7}$ & $\underline{16,8}$ & $\underline{74,5}$ \\
\hline $\begin{array}{l}\text { Pensez-vous que la police de type } \\
\text { communautaire a fait augmenter, } \\
\text { diminuer ou n'a eu aucun impact sur le } \\
\text { nombre d'incidents impliquant une force } \\
\text { excessive de la part de policiers? }\end{array}$ & $\underline{1,5}$ & $\underline{28,3}$ & $\underline{70,1}$ \\
\hline $\begin{array}{l}\text { Pensez-vous que la police de type } \\
\text { communautaire a fait augmenter, } \\
\text { diminuer ou n'a eu aucun impact sur la } \\
\text { gravité des incidents impliquant une } \\
\text { force excessive de la part des policiers? }\end{array}$ & $\underline{1,9}$ & $\underline{27,0}$ & $\underline{71,1}$ \\
\hline
\end{tabular}

que les répondants italiens et étatsuniens sont sur une même longueur d'onde sur le plan des principes éthiques et organisationnels susceptibles de permettre un certain encadrement de l'abus d'autorité et des conduites policières malsaines. Par contre, les répondants italiens se démarquent quelque peu des répondants étatsuniens en affichant un certain scepticisme quant à la volonté concrète de leur organisation de passer à l'action à ce sujet, bien que, d'un autre côté, les principes au nom desquels une hiérarchie doit intervenir dépassent sensiblement la notion de solidarité entre collègues. Dans la section suivante, nous nous attarderons plus précisément à des questions qui illustrent le positionnement éthique des répondants; il conviendra alors de vérifier jusqu’à quel point les différences relevées jusqu'ici vont également ressortir de la prochaine série d'analyses, notamment sur la question des différences observées quant à la distance entre le raisonnement personnel, et celui que l'on attribue à son organisation et à ses collègues.

\section{Éthique, sanctions et dénonciation}

Le tableau suivant énonce les 11 scénarios, ou vignettes, à propos desquels les répondants ont consigne de se prononcer. 
TABLEAU 7

Les onze scénarios proposés par le questionnaire de Klockars et al. (1997)

\begin{tabular}{|c|c|}
\hline Numéros & Texte des scénarios \\
\hline 1 & $\begin{array}{l}\text { Un policier exploite sa propre entreprise de vente et d'installation de } \\
\text { matériel de sécurité tel que systèmes d'alarme, serrures spéciales, etc. Il } \\
\text { effectue ce travail durant ses heures libres. }\end{array}$ \\
\hline 2 & $\begin{array}{l}\text { Un policier accepte régulièrement repas gratuits, cigarettes et autres } \\
\text { items de peu de valeur des marchands de son secteur de patrouille. Il ne } \\
\text { demande pas ces dons et s'assure de ne pas abuser de la générosité de } \\
\text { ceux qui lui font ces cadeaux. }\end{array}$ \\
\hline 3 & $\begin{array}{l}\text { Un policier de la route interpelle un conducteur pour excès de vitesse. Le } \\
\text { policier accepte un don en argent qui représente la moitié de la contra- } \\
\text { vention et, en échange, il n'émet pas ladite contravention. }\end{array}$ \\
\hline 4 & $\begin{array}{l}\text { Un policier est bien aimé dans la communauté et durant la période de } \\
\text { Noël, les marchands locaux, les propriétaires de restaurants et de bars lui } \\
\text { montrent leur appréciation pour son dévouement sous forme de dons de } \\
\text { nourriture et alcools. }\end{array}$ \\
\hline 5 & $\begin{array}{l}\text { Un policier découvre une introduction par effraction dans une bijouterie. } \\
\text { Les vitres des présentoirs sont brisées et il est apparent que plusieurs items } \\
\text { ont été dérobés. Alors qu'il enquête sur les lieux, il subtilise une montre } \\
\text { dont la valeur équivaut à deux jours de son salaire. Il inclut la montre dans } \\
\text { la liste des items qui ont été volés lors de l'introduction par effraction. }\end{array}$ \\
\hline 6 & $\begin{array}{l}\text { Un policier a une entente avec un carrossier. Il lui adresse les propriétai- } \\
\text { res de véhicules endommagés lors d'accidents routiers sur lesquels il } \\
\text { enquête. Il touche } 5 \% \text { de la facture de réparation en commission de la } \\
\text { part du carrossier. }\end{array}$ \\
\hline 7 & $\begin{array}{l}\text { Un policier est également un excellent mécanicien. Il est prévu qu'il travaille } \\
\text { durant la période de Noël qui approche. Son superviseur lui offre de lui } \\
\text { accorder plutôt ce congé s'il accepte de faire la mise au point de la voiture } \\
\text { personnelle du superviseur. Évaluez le comportement du superviseur. }\end{array}$ \\
\hline 8 & $\begin{array}{l}\text { À } 2 \text { h } 00 \text { du matin, un policier de service conduit son auto-patrouille sur } \\
\text { une route déserte. Il aperçoit un véhicule qui a fait une sortie de route } \\
\text { et qui est embourbé dans un fossé. Il s'approche du véhicule et constate } \\
\text { que le conducteur n'est pas blessé, mais a de toute évidence les facultés } \\
\text { affaiblies par l'alcool. Il constate aussi que le conducteur est un policier. } \\
\text { Plutôt que de rapporter l'offense, il reconduit le conducteur chez lui. }\end{array}$ \\
\hline 9 & $\begin{array}{l}\text { Un policier trouve un bar dans son secteur de patrouille qui sert encore } \\
\text { de l'alcool une demi-heure après l'heure de fermeture obligatoire. Plutôt } \\
\text { que de rapporter cette infraction, le policier accepte quelques verres de } \\
\text { la part du tenancier. }\end{array}$ \\
\hline 10 & $\begin{array}{l}\text { Deux patrouilleurs à pied surprennent un homme qui tente de s'introduire } \\
\text { dans un véhicule. L'homme s'enfuit. Les deux policiers le poursuivent sur } \\
\text { deux pâtés de maisons et le rattrapent en le jetant par terre. Une fois le } \\
\text { suspect sous contrôle, ils le tabassent de quelques bons coups de poing } \\
\text { à l'abdomen en guise de punition pour avoir fui. }\end{array}$ \\
\hline 11 & $\begin{array}{l}\text { Un policier découvre un portefeuille dans un stationnement. Le porte- } \\
\text { feuille contient l'équivalent d'une journée de salaire du policier. Le policier } \\
\text { rapporte le portefeuille comme objet trouvé mais garde l'argent. }\end{array}$ \\
\hline
\end{tabular}


TABLEA U 8

Sommaire comparé des réponses aux 11 scénarios du sondage de Klockars et al. (1997) entre l'échantillon original étatsunien (en caractères normaux) et l'échantillon italien ${ }^{a}$ (en caractères soulignés)

\begin{tabular}{|c|c|c|c|c|c|c|c|c|c|c|c|c|c|}
\hline \multirow{3}{*}{\multicolumn{2}{|c|}{ Scénario }} & \multicolumn{4}{|c|}{ Gravité } & \multicolumn{4}{|c|}{ Mesure disciplinaire } & \multicolumn{4}{|c|}{ Prédisposition à dénoncer } \\
\hline & & \multicolumn{2}{|c|}{ Eux-mêmes } & \multicolumn{2}{|c|}{ Les autres } & \multicolumn{2}{|c|}{ Devrait recevoir } & \multicolumn{2}{|c|}{ Reçoit réellement } & \multicolumn{2}{|c|}{ Eux-mêmes } & \multicolumn{2}{|c|}{ Les autres } \\
\hline & & Moyenne & Rang & Moyenne & Rang & Moyenne & Rang & Moyenne & Rang & Moyenne & Rang & Moyenne & Rang \\
\hline \multirow{2}{*}{$\begin{array}{l}\text { 1. Entreprise de } \\
\text { systèmes de sécurité, } \\
\text { hors des heures } \\
\text { de travail. }\end{array}$} & EU & 1,46 & 1 & 1,48 & 1 & 1,34 & 1 & 1,51 & 1 & 1,37 & 1 & 1,46 & 1 \\
\hline & Ital & $\underline{2,86}$ & $\underline{2}$ & $\underline{2,58}$ & $\underline{2}$ & $\underline{2,30}$ & $\underline{2}$ & $\underline{2,27}$ & $\underline{2}$ & $\underline{1,89}$ & $\underline{1}$ & $\underline{1,74}$ & $\underline{2}$ \\
\hline \multirow{2}{*}{$\begin{array}{l}\text { 2. Repas gratuit et } \\
\text { rabais pendant le } \\
\text { quart de travail. }\end{array}$} & EU & 2,60 & 2 & 2,31 & 2 & 2,13 & 2 & 2,37 & 2 & 1,94 & 2 & 1,82 & 2 \\
\hline & Ital & $\underline{3,76}$ & $\underline{4}$ & $\underline{3,42}$ & $\underline{4}$ & $\underline{2,68}$ & $\underline{3}$ & $\underline{2,54}$ & $\underline{3}$ & $\underline{2,34}$ & $\underline{3}$ & $\underline{2,10}$ & $\underline{3}$ \\
\hline \multirow{2}{*}{$\begin{array}{l}\text { 3. Accepter un pot- } \\
\text { de-vin de la part } \\
\text { d'un automobiliste } \\
\text { intercepté pour excès } \\
\text { de vitesse. }\end{array}$} & EU & 4,92 & 10 & 4,81 & 10 & 4,92 & 9 & 4,86 & 9 & 4,19 & 9 & 3,92 & 9 \\
\hline & Ital & $\underline{4,93}$ & $\underline{11}$ & $\underline{4,86}$ & $\underline{11}$ & $\underline{4,99}$ & $\underline{10}$ & $\underline{4,72}$ & $\underline{10}$ & 4,07 & $\underline{10}$ & $\underline{3,70}$ & $\underline{10}$ \\
\hline \multirow{2}{*}{$\begin{array}{l}\text { 4. Cadeaux des } \\
\text { commerçants lors de } \\
\text { la période de Noël. }\end{array}$} & EU & 2,84 & 3 & 2,64 & 3 & 2,54 & 3 & 2,82 & 3 & 2,36 & 4 & 2,28 & 3,5 \\
\hline & Ital & $\underline{2,59}$ & $\underline{1}$ & $\underline{2,40}$ & $\underline{1}$ & $\underline{1,75}$ & $\underline{1}$ & $\underline{1,76}$ & $\underline{1}$ & $\underline{3,62}$ & $\underline{7}$ & $\underline{1,54}$ & $\underline{1}$ \\
\hline \multirow{2}{*}{$\begin{array}{l}\text { 5. Vol d'une montre sur } \\
\text { une scène de crime. }\end{array}$} & EU & 4,95 & 11 & 4,88 & 11 & 5,66 & 11 & 5,57 & 11 & 4,54 & 11 & 4,34 & 11 \\
\hline & Ital & $\underline{4,88}$ & $\underline{10}$ & $\underline{4,82}$ & $\underline{10}$ & $\underline{5,11}$ & $\underline{11}$ & $\underline{4,97}$ & $\underline{11}$ & $\underline{4,10}$ & $\underline{11}$ & $\underline{3,76}$ & $\underline{11}$ \\
\hline \multirow{2}{*}{$\begin{array}{l}\text { 6. } 5 \% \text { de ristourne pour } \\
\text { référence à un garage. }\end{array}$} & EU & 4,50 & 7 & 4,26 & 7 & 4,40 & 8 & 4,46 & 8 & 3,95 & 8 & 3,71 & 8 \\
\hline & Ital & $\underline{4,76}$ & $\underline{9}$ & $\underline{4,59}$ & $\underline{9}$ & $\underline{4,61}$ & $\underline{9}$ & $\underline{4,39}$ & $\underline{9}$ & $\underline{3,86}$ & $\underline{9}$ & $\underline{3,49}$ & $\underline{9}$ \\
\hline
\end{tabular}


TABLEAU 8 (suite)

\begin{tabular}{|c|c|c|c|c|c|c|c|c|c|c|c|c|c|}
\hline \multirow{3}{*}{\multicolumn{2}{|c|}{ Scénario }} & \multicolumn{4}{|c|}{ Gravité } & \multicolumn{4}{|c|}{ Mesure disciplinaire } & \multicolumn{4}{|c|}{ Prédisposition à dénoncer } \\
\hline & & \multicolumn{2}{|c|}{ Eux-mêmes } & \multicolumn{2}{|c|}{ Les autres } & \multicolumn{2}{|c|}{ Devrait recevoir } & \multicolumn{2}{|c|}{ Reçoit réellement } & \multicolumn{2}{|c|}{ Eux-mêmes } & \multicolumn{2}{|c|}{ Les autres } \\
\hline & & Moyenne & Rang & Moyenne & Rang & Moyenne & Rang & Moyenne & Rang & Moyenne & Rang & Moyenne & Rang \\
\hline \multirow{2}{*}{$\begin{array}{l}\text { 7. Un superviseur qui } \\
\text { accorde un congé } \\
\text { contre une mise au } \\
\text { point par un des } \\
\text { policiers dont il est } \\
\text { responsable. }\end{array}$} & EU & 4,18 & 6 & 3,96 & 6 & 3,59 & 5 & 3,43 & 5 & 3,45 & 6 & 3,29 & 6 \\
\hline & Ital & $\underline{4,53}$ & $\underline{7}$ & $\underline{4,36}$ & $\underline{7}$ & $\underline{3,41}$ & $\underline{7}$ & $\underline{2,91}$ & $\underline{6}$ & $\underline{3,23}$ & $\underline{6}$ & $\underline{2,95}$ & $\underline{7}$ \\
\hline \multirow{2}{*}{$\begin{array}{l}\text { 8. Couvrir un policier } \\
\text { pour ivresse au } \\
\text { volant. }\end{array}$} & EU & 3,03 & 4 & 2,86 & 4 & 2,81 & 4 & 3,21 & 4 & 2,34 & 3 & 2,28 & 3,5 \\
\hline & Ital & $\underline{3,39}$ & $\underline{3}$ & $\underline{3,16}$ & $\underline{3}$ & $\underline{2,76}$ & $\underline{4}$ & $\underline{2,66}$ & $\underline{4}$ & $\underline{2,27}$ & $\underline{2}$ & $\underline{2,12}$ & $\underline{4}$ \\
\hline \multirow{2}{*}{$\begin{array}{l}\text { 9. Accepter des } \\
\text { consommations pour } \\
\text { ignorer l'heure de } \\
\text { fermeture d'un bar. }\end{array}$} & EU & 4,54 & 8 & 4,28 & 8 & 4,02 & 7 & 4,08 & 7 & 3,73 & 7 & 3,47 & 7 \\
\hline & Ital & $\underline{4,28}$ & $\underline{6}$ & $\underline{4,05}$ & $\underline{6}$ & $\underline{3,00}$ & $\underline{5}$ & $\underline{2,85}$ & $\underline{5}$ & $\underline{2,90}$ & $\underline{4}$ & $\underline{2,66}$ & $\underline{6}$ \\
\hline \multirow{2}{*}{$\begin{array}{l}\text { 10. Utilisation de force } \\
\text { excessive à l'endroit } \\
\text { d'un suspect lors } \\
\text { d'une tentative de } \\
\text { vol de véhicule. }\end{array}$} & EU & 4,05 & 5 & 3,70 & 5 & 3,76 & 6 & 4,00 & 6 & 3,39 & 5 & 3,07 & 5 \\
\hline & Ital & $\underline{3,98}$ & $\underline{5}$ & $\underline{3,69}$ & $\underline{5}$ & $\underline{3,26}$ & $\underline{6}$ & $\underline{3,13}$ & $\underline{7}$ & $\underline{2,92}$ & $\underline{5}$ & $\underline{2,62}$ & $\underline{5}$ \\
\hline \multirow{2}{*}{$\begin{array}{l}\text { 11. Vol dans un } \\
\text { porte-monnaie } \\
\text { trouvé. }\end{array}$} & EU & 4,85 & 9 & 4,69 & 9 & 5,09 & 10 & 5,03 & 10 & 4,23 & 10 & 3,96 & 10 \\
\hline & Ital & $\underline{4,74}$ & $\underline{8}$ & $\underline{4,57}$ & $\underline{8}$ & $\underline{4,35}$ & $\underline{8}$ & $\underline{4,16}$ & $\underline{8}$ & $\underline{3,78}$ & $\underline{8}$ & $\underline{3,48}$ & $\underline{8}$ \\
\hline
\end{tabular}

a: les paramètres de l'échantillon italien ont été pondérés pour tenir compte de la distribution des grades dans la population. 
Comme nous l'avons brièvement mentionné en introduction, ce questionnaire a déjà fait l'objet de plusieurs passations dans pas moins de 14 pays aux traditions et aux systèmes policiers très différents les uns des autres. Dans le cas qui nous concerne, nous comparerons les réponses données par deux échantillons, soit celui de l'étude étatsunienne d'origine et celui de la région toscane. Le tableau suivant fait état des statistiques descriptives et du rang occupé par les onze vignettes, en fonction des thématiques abordées dans le questionnaire.

Un premier coup d'œil, plus global, aux éléments contenus dans ce tableau, et si l'on s'en tient aux rangs de gravité des onze vignettes, révèle quelques différences importantes sur le plan des opinions personnelles. Ainsi, les scénarios 2 (repas gratuit), 4 (cadeaux des commerçants), 6 ( $5 \%$ de ristourne pour adresser des clients à un garage) et 9 (accepter des consommations) affichent au moins deux rangs d'écart dans les réponses données par les deux échantillons. Les scénarios 2 et 6 sont jugés plus graves par les répondants italiens que par les répondants étatsuniens. C'est le contraire pour ce qui est des scénarios 4 et 9. Nul doute, ici, que ces différences sont intimement liées à des contingences d'intervention (Manning, 2003; Terril et Mastrofski, 2002; Wellford et al., 2002) probablement assez différentes d'un contexte à l'autre. Dans le cas de l'Italie, par exemple, une tradition était en vigueur jusqu'à il n'y a que quelques années, tradition dite de la «Befana del Vigile», et voulant qu'à l'occasion de la fête de l'Épiphanie, les commerçants, entreprises et citoyens fassent des cadeaux en boisson et nourriture à leurs policiers pour les remercier et reconnaitre leur engagement dans la communauté.

Mais si, donc, des différences verticales apparaissent, en revanche, on observera des éléments de cohésion culturelle importants qui transcendent les contextes d'intervention; dans ce cas-ci, il conviendra alors de faire allusion à des similarités horizontales. Cette fois-ci, plutôt que de nous livrer au fastidieux exercice de comparaison d'une case avec une autre dans un tableau qui demeure, nous l'admettons, assez indigeste, nous privilégierons des analyses un peu plus statistiques. Quelques graphiques nous permettront d'abord d'établir une base comparative avec les réponses données par les répondants étatsuniens. Comme nous ne possédons pas les données originales de cette étude, le traitement graphique s'impose un peu de lui-même. 
F I G URE 1

Niveau de gravité des scénarios (échantillon étatsunien)

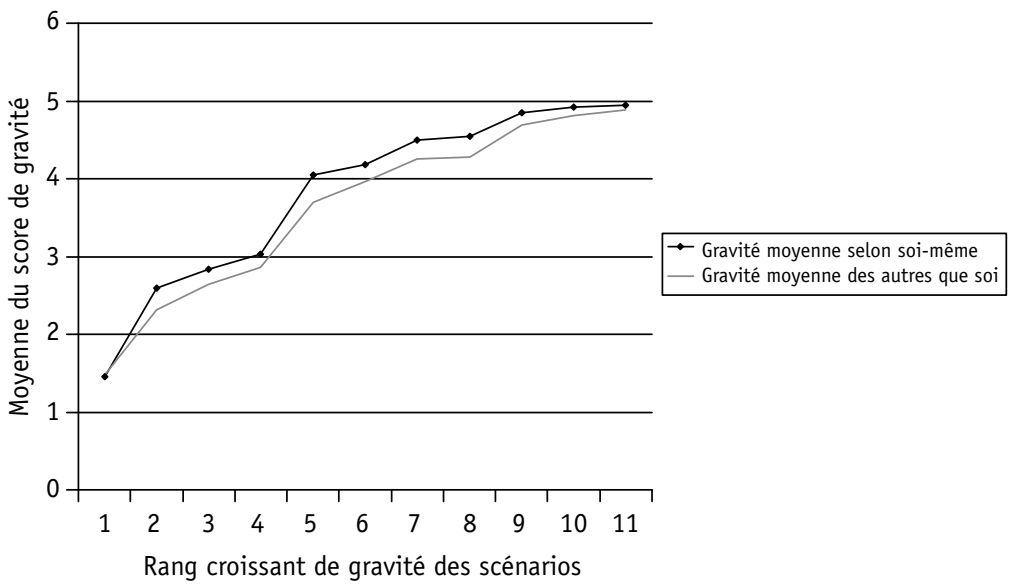

On observe, à partir des courbes illustrées au graphique précédent, une très grande cohésion entre, d'une part, ce que le répondant pense de la gravité des comportements décrits dans chacun des scénarios et, d'autre part, le jugement qu'il attribue à ses collègues: le jugement personnel et parfaitement aligné sur celui que l'on attribue à ses pairs. Mais, mis à part le cas des scénarios jugés aux deux extrêmes de l'échelle de gravité (soit les scénarios 1 et 5), on observe aussi un certain décalage entre les deux courbes. Ce décalage illustre en fait que le répondant pense qu'il est lui-même plus sévère que ses collègues. Le graphique suivant illustre cette comparaison, mais cette fois-ci sur le plan des mesures disciplinaires que le répondant entendrait donner à un policier reconnu coupable du comportement décrit dans les vignettes et, d'autre part, ce qu'il pense de la manière dont son organisation sanctionnerait le comportement en question.

Premier élément à ressortir de ce graphique, on remarquera que, mis à part le cas du scénario placé au cinquième rang, le décalage entre la sanction privilégiée par le répondant et celle qu'il pense que son organisation donnerait tend à s'amenuiser au fur et à mesure que le rang de sévérité s'accroît. C'est dire, en d'autres termes, que dans le cas des quatre premiers rangs, le répondant tend à juger la réponse attendue de son organisation comme plus dure que celle que lui-même donnerait; par contre, à partir du septième rang, cette distance s'estompe et les 


\section{F I G URE 2}

Mesures disciplinaires en fonction de la perception du degré de gravité de l'acte posé dans les scénarios (échantillon étatsunien)

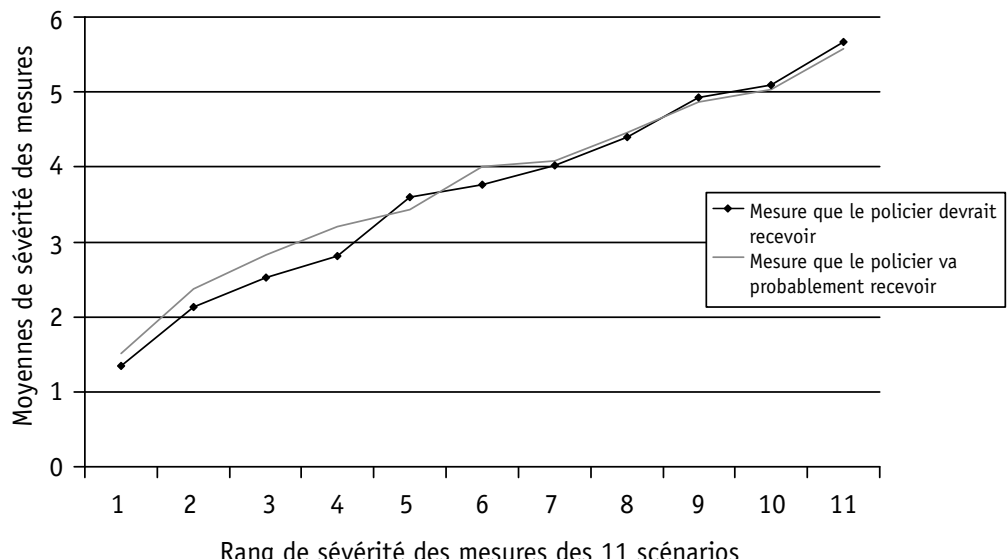

deux courbes se confondent. Dernier thème à discuter pour ce qui est d'une analyse horizontale des réponses de l'échantillon original étatsunien: la question de la propension à dénoncer ou non les comportements décrits dans les vignettes, objet de l'illustration suivante.

\section{F I G U RE 3}

La prédisposition à dénoncer les comportements décrits (échantillon étatsunien)

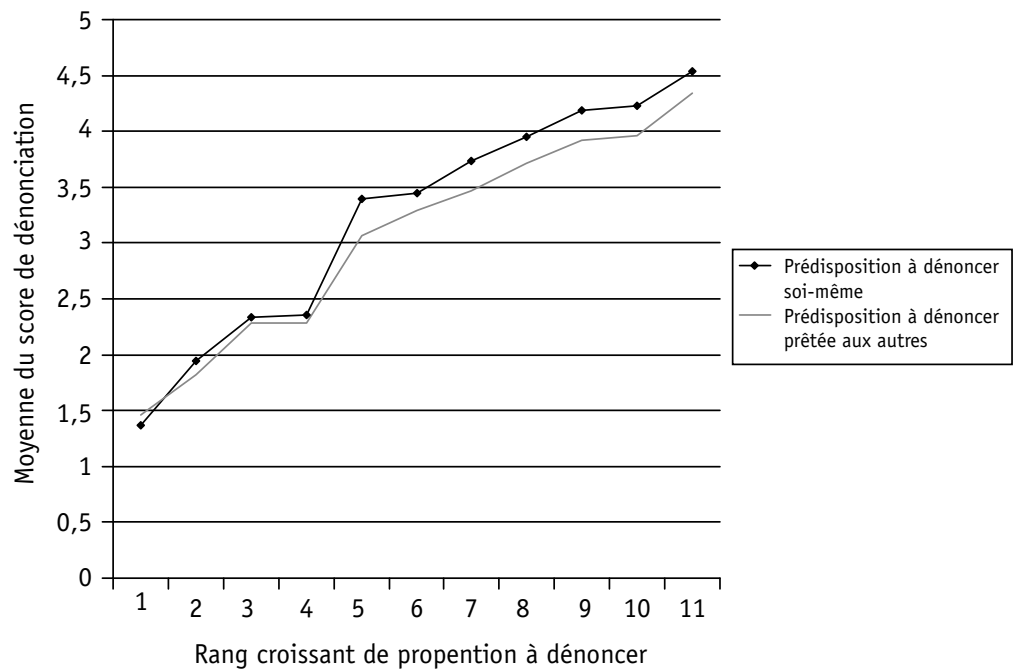


On observe une tendance quelque peu opposée à ce que nous avons analysé au thème précédent: si la position personnelle du répondant dans sa velléité à dénoncer est calquée sur celle qu'il prête à ses collègues dans le cas des quatre premiers scénarios, à partir du cinquième, le répondant pense de lui-même qu'il est plus prompt à dénoncer que ses collègues. Nous avons donc essentiellement trois choses à vérifier sur le plan des réponses fournies par l'échantillon de policiers italiens: (1) ont-ils l'impression que leurs collègues jugent les comportements moins sévèrement qu'eux-mêmes? (2) sont-ils un peu moins sévères que ce qu'ils croient de leur hiérarchie? (3) pensent-ils être plus prompts à dénoncer leurs collègues? Ces trois questions font l'objet du tableau suivant.

TABLEAU 9

Comparaison entre les opinions personnelles des répondants et ce que ces mêmes répondants prêtent à leurs collègues - ou à leur organisation - en termes de sévérité de la sanction; moyennes sur les 11 scénarios proposés ${ }^{a}$

\begin{tabular}{|l|c|c|c|c|c|}
\hline & \multicolumn{2}{|c|}{$\begin{array}{c}\text { Opinions } \\
\text { personnelles }\end{array}$} & \multicolumn{2}{c|}{$\begin{array}{c}\text { Opinions prêtées } \\
\text { aux autres } \\
\text { (à l'organisation) }\end{array}$} & \\
\hline Variables & $M$ & ÉT & $M$ & ÉT & $t$ \\
\hline $\begin{array}{l}\text { Gravité des scénarios } \\
(n=790)\end{array}$ & 4,05 & 0,52 & 3,82 & 0,58 & $15,96^{* * *}$ \\
$\begin{array}{l}\text { Sévérité de la sanction } \\
(n=738)\end{array}$ & 3,47 & 0,67 & 3,29 & 0,78 & $7,90^{* * *}$ \\
$\begin{array}{l}\text { Prédisposition à dénoncer } \\
(n=718)\end{array}$ & 3,01 & 0,90 & 2,73 & 0,85 & $12,99 * * *$ \\
\hline
\end{tabular}

${ }^{a}$ : l'échantillon italien est pondéré en fonction des grades de manière à l'apparier à la population dont il est tiré. ${ }^{* \star} p<0,001$.

Nous attirons tout d'abord l'attention du lecteur sur la dernière colonne du tableau 9, soit celle qui fait mention de la statistique $t$ des différences de moyenne. Nous avons ici traité de ces différences en recourant à un simple test $\mathrm{t}$ de Student sur échantillons appariés. Dans les trois cas, ces différences sont statistiquement significatives et elles confirment, comme dans le cas de l'échantillon étatsunien, des différences entre son opinion et la perception que le répondant a de l'opinion des autres et de son organisation. Or, si, dans le premier et le troisième thème (gravité des scénarios et prédisposition à dénoncer), les différences observées vont dans le même sens pour les deux échantillons, ce 
n'est pas le cas du second thème (celui lié à la notion de sévérité des sanctions): dans ce cas-ci, les répondants italiens pensent que leur organisation serait moins sévère qu'eux-mêmes auraient tendance à l'être.

On ne peut faire autrement, dans ce dernier cas, que de faire un lien entre les réponses des répondants italiens aux deux questionnaires. On se rappellera, en effet, sur le plan des conduites organisationnelles susceptibles de contrôler l'abus d'autorité (voir tableau 5), et celui, plus général, de l'impact positif du modèle de police communautaire (voir tableau 6), que les répondants italiens expriment une opinion plus réservée que celle de leurs collègues étatsuniens. C'est dire, en d'autres termes, que les répondants italiens font montre d'une adhésion aux principes éthiques - les leurs et ceux que l'on prête à la hiérarchie sensiblement plus importante que celle démontrée par les répondants étatsuniens. En revanche, les répondants italiens sont plus sceptiques que leurs collègues étatsuniens quant à la mise en place réelle et mesurable de politiques et de mesures efficaces à cet effet. Et ici, force nous est de penser que ces opinions se trouvent malheureusement appuyées par les difficultés réelles de l'Italie à sanctionner des policiers ou des sections d'organisations policières fautives tel que nous l'avons décrit en première section de cet article.

\section{Conclusion}

La question globale de la recherche d'une certaine homogénéité culturelle policière pourra, au terme de notre exercice, faire l'objet de plusieurs nuances. En ce sens, nos données et les discussions qu'elles permettent nous montrent que sur des thématiques liées aux différentes cultures organisationnelles policières - i. e. objets traités dans le premier bloc de données, celles liées à la réplique des travaux de Weisburd et al. 2000 - des divergences apparaissent mais globalement; nous avons vu que les répondants des deux échantillons s'en tiennent à des opinions qui évoquent une vision quelque peu idéalisée de la police, ce qui nous amenait même à évoquer la notion d'hétéroduperie. Les répondants italiens, en revanche, et on l'a observé autant dans le premier bloc de données que dans le second (i. e. les questions liées aux onze vignettes de comportement dérogatoires), ont tendance à être un peu plus sceptiques que leurs collègues quant à la possibilité que leur organisation soit prompte à punir les policiers fautifs. Le second bloc de données, 
cependant, nous a donné l'occasion de constater de manière globale à quel point les opinions des policiers à l'égard de la gravité des comportements décrits dans les vignettes de Klockars et al. sont très semblables d'un échantillon à l'autre, une observation que faisaient également Klockars et ses collègues dans leurs travaux touchant 14 pays différents. Là encore, toutefois, les répondants italiens se démarquent quelque peu, et c'est encore une fois sur le plan de leur perception des velléités organisationnelles à contrôler les comportements problématiques. La question de savoir, maintenant, comment il se fait que le répondant émet une opinion éthique toujours mieux située que celle qu'il attribue à ses collègues, et ce, dans les deux échantillons, constitue une piste qu'il conviendrait d'explorer plus avant, ne serait-ce, par exemple, qu'en allant investiguer d'autres univers professionnels ${ }^{12}$.

Finalement, et nous conclurons sur cette note, la question du scepticisme exprimé par les répondants de la région toscane sur la volonté de leur organisation de mieux contrôler les abus pourrait, jusqu'à un certain point, être utilisable à court terme. En effet, l'exercice auquel nous nous sommes livrés avec cette population a été non seulement permis, mais même encouragé par les instances administratives de la région toscane. Ces instances avaient alors comme projet d'introduire certains changements importants dans la prestation des services policiers dont elles assument la responsabilité. Les éléments révélés dans l'analyse des réponses que nous ont données leurs employés devraient être tenus en compte au moment où ces changements feront l'objet de discussions et d'opérationnalisation. Dans le cas contraire, des résistances internes risquent d'être opposées à ces réformes.

12. Klockars et al. (2004: 10) ne sont d'ailleurs guère plus explicites quant à une explication potentielle à l'égard de cette différence entre l'opinion personnelle du répondant et celle qu'il prête à ses collègues: "While most officers can imagine that they are morally superior to some officers or that some officers are their moral inferiors, to claim that they are substantially morally superior to most officers is a claim that most officers are not willing to make.» Peut-être, et ce n'est là tout au plus qu'une idée à discuter, pourrions-nous remonter au texte classique de John Van Maanen (1978), The Asshole, où celui-ci traitait, avec une sérieuse dose d'irrévérence, du sentiment de moralité supérieure de l'officier de police, non seulement à l'égard de certains citoyens mais qui plus est, de certains de ses propres collègues au sein de son organisation. 


\section{Références}

Alain, M. (2000). Les heurts et les bonheurs de la coopération policière internationale en Europe, entre la myopie des bureaucrates et la sclérose culturelle policière. Déviance et Société, 24 (3), 237-253.

Alain, M. (2001). 'The trapeze artists and the ground crew'; police cooperation and intelligence exchange mechanisms in Europe and in North America, a comparative empirical study. Policing $\mathbb{W}$ Society, 11 (1), 1-27.

Alain, M. (2004). Une mesure de la propension des policiers québécois à dénoncer des comportements dérogatoires, éléments de culture policière et cultures organisationnelles. Déviance et Société, 28 (1), 2-32.

Alain, M., \& Grégoire, M. (2008). Can ethics survive the shock of the job? Quebec's police recruits confront reality. Policing $\mathcal{W}$ Society, 18 (2), 169-189.

Bittner, E. (1967a). The police on skid row: A study of peacekeeping. American Sociological Review, 32 (5), 699-715.

Bittner, E. (1967b). Police discretion in the apprehension of mentally ill persons? Social Problems, 14 (3), 278-292.

Bittner E., \& Platt A. M. (1966). The meaning of punishment. Issues in Criminology, 2 (1), 77-99.

Carrer, F. (2004). Insécurité urbaine et politiques d'interventions: la réalité italienne. Déviance et Société, 28 (4), 463-485.

Carrer, F. (2006). The Italian Police Forces and the Internal Security System. Crime $\mathfrak{*}$ justice international, 92, 11-17.

Greene, J. R., Bergman, W. T., \& McLaughlin, E. J. (1994). Implementing Community Policing: Cultural and Structural Change in Police Organizations. In D. P. Rosenbaum (Ed.), The Challenge of Community Policing (92-109). Thousand Oaks: Sage.

Ivkovic, S. K., \& Klockars, C. B. (1995). Cross-Cultural Study of Police Corruption: Perceptions of Offense Seriousness; Police Perceptions of Disciplinary Fairness and Code of Silence. United States: Bureau of Justice Assistance.

Klockars, C. B., Ivkovic S. K., \& Haberfeld M. R. (2003) The Contours of Police Integrity. Thousand Oakes: Sage.

Klockars, C. B., Ivkovic, S. K., Harver, W. E., Haberfeld, M. R. (1997). Measurement of Police Integrity, Final Report. United States: National Institute of Justice.

Knapp, W. (1972). The Knapp Commission Report on Police Corruption. New York: Georges Braziller.

Manning, P. K. (2003). Policing Contingencies. Chicago: The University of Chicago Press.

Macpherson, W. (1999). The Stephen Lawrence Inquiry - Report of an Inquiry. Presented to Parliament by the Secretary of State for the Home Department by Command of Her Majesty, February. London.

Mollen, M. (1994). Commission report: commission to investigate allegations of police corruption and the anti-corruption procedures of the police department. New York: The City of New-York. 
O'Loan, N. (2001). Report into the Investigation of Matters relating to the Omagh Bomb on 15 August 1998. Belfast: Police Ombudsman for Northern Ireland.

Patten, C. F. (1999). A New Beginning: Policing in Northern Ireland. Belfast: Independent Commission On Policing for Northern Ireland.

Punch, M., Huberts, L., \& Lamboo, T. (2000). Perceptions on Integrity of Dutch Police Officers in Comparative Perspective. IIPE Conference on Ethics in the New Millenium. Ottawa.

Rokeach, M., Miller, M., \& Snyder, J. (1971). The value gap between police and policed. Journal of Social Issues, 27 (2), 155-171.

Scarman, L. G. (1982). The Scarman Report: The Brixton Disorders 10-12 April 1981 : Report of an Inquiry. Harmondsworth: Penguin.

Sudman, S. (1976). Applied Sampling. New York: Academic Press.

Terrill, W., \& Mastrofski, S. D. (2002). Situational and Officer-based Determinants of Police Coercion. Justice Quarterly, 19 (2), 215-248.

Tournois J., Mesnil, F., \& Kop, J.-L. (2000). Autoduperie et hétéroduperie: Un instrument de mesure de la désirabilité sociale. Paris: Centre de psychologie appliqué.

Van Maanen, J. (1978). The Asshole. In P. K. Manning \& J. Van Maanen (Eds.), Policing, A View from the Street (221-238). New York: Random House.

Weisburd, D., Greenspan, R., Hamilton, E. E., Bryant, K. A., \& Williams, H. (2001). The abuse of police authority: a National Study of Police Officer's Attitude. Washington: Police Foundation.

Weisburd, D., Greenspan, R., Hamilton, E. E., Williams, H, \& Bryant, K. A. (2000). Police Attitudes Toward Abuse of Authority: Findings From a National Study. Washington: Police Foundation.

Wellford, W.W., Schmidt, W. H., \& Norman, A. J. (2002). "To Protect and to Serve... and To Listen". Adding a new dimension to policing Los Angeles. Les Angeles: University of California.

ABSTRACT - This article, empirical and descriptive in its nature, presents an overview of the Italian policing system and the context of policing in the northern region of Tuscan. Thereafter, we present the results obtained from a survey that was conducted among 1520 police officers from this region. The survey tool combines the questionnaire designed by Weisburd et al. (2000), where the questions are aimed at common police organisational culture themes, and the questionnaire designed separately by Klockars et al. (1997) in order to assess the ethical position of police officers regarding 11 vignettes of derogatory conducts. The results from the Tuscan region are then compared to the two original US studies. This comparison yields to elements of what has been referred in the literature as a general police culture, as well as elements that tend to indicate dissimilarities that we attribute to important contextual differences in the policing systems of the two countries.

KEYWORDS - Police ethics, police professional culture, police organizations' management, intercultural comparisons. 
RESUMEN - Este artículo presenta una descripción general del funcionamiento de la policía en Italia y sitúa la correspondiente a la región de Toscana. A continuación se presentan los resultados de la colecta de datos de 1520 policías de dicha organización que aceptaron responder un cuestionario anónimo destinado a medir, por una parte, el grado de apego a cinco grandes temas de la cultura profesional policiaca y, por otra parte, la propensión a denunciar o no comportamientos derogatorios hipotéticos presentados en forma de viñetas. Los resultados obtenidos con la policía de Toscana se comparan en seguida con los obtenidos en los cuestionarios originales aplicados en dos estudios estadounidenses. La comparación permite analizar las especificidades italianas, lo mismo que algunas características que podemos atribuir a la cultura profesional policiaca en general.

PALABRAS CLAVE - Ética policiaca, cultura profesional policiaca, administración de una organización policiaca, comparaciones interculturales. 